\title{
The Clinical and Economic Impact of Alternative Staging Strategies for Adenocarcinoma of the Pancreas
}

\author{
William M. Tierney, M.D., A. Mark Fendrick, M.D., Richard A. Hirth, Ph.D., and James M. Scheiman, M.D. \\ Division of Gastroenterology, Department of Internal Medicine, University of Michigan School of Medicine; \\ Consortium of Health Outcomes, Innovation, and Cost-effectiveness Studies (CHOICES), University of \\ Michigan School of Medicine; Division of General Medicine, Department of Internal Medicine, University of \\ Michigan School of Medicine, Ann Arbor, Michigan; and Department of Health Management and Policy, \\ University of Michigan School of Public Health, Ann Arbor, Michigan
}

OBJECTIVE: Several innovative imaging modalities, including endoscopic ultrasound, have increased the number of available preoperative staging methods in patients with adenocarcinoma of the pancreas. Our goal was to estimate the clinical outcomes and cost-effectiveness of alternative staging strategies for pancreatic adenocarcinoma.

METHODS: Decision analysis was used to simulate alternative staging strategies. Cost inputs were based on Medicare reimbursements; clinical inputs were obtained from the available literature. Model endpoints of interest were cost per curative resection and appropriateness of treatment allocation based on pathological stage.

RESULTS: Endoscopic ultrasound followed by laparoscopy yielded the lowest cost per curative resection $(\$ 37,600)$ and minimized the number of unnecessary surgical explorations (5.4 per 100 patients staged). Requiring angiographic confirmation when endoscopic ultrasound demonstrated an unresectable tumor yielded an intermediate cost-effectiveness ratio and virtually eliminated the risk of overstaging. Laparoscopy alone maximized the resection rate, but each additional resection would cost approximately $\$ 2$ million relative to a strategy employing both endoscopic ultrasound and angiography.

CONCLUSIONS: Staging strategies incorporating endoscopic ultrasound may improve treatment allocation and are costeffective relative to angiography-based strategies. A staging protocol that does not incorporate an imaging modality to detect vascular invasion dramatically increases the cost per additional curative resection compared with more comprehensive staging protocols. (Am J Gastroenterol 2000;95: 1708-1713. (C) 2000 by Am. Coll. of Gastroenterology)

Presented in part at the Annual Meeting of the American Gastroenterology Association (1996) San Francisco, CA.

\section{INTRODUCTION}

Adenocarcinoma of the pancreas is the fifth leading cause of cancer death in the United States and approximately 27,000 new cases are diagnosed annually (1). Disease is usually advanced at the time of diagnosis and only $15 \%$ of patients have localized tumors amenable to attempted curative resection (2-7). The recent reports of 5-yr postresection survival rates of $>20 \%$ and surgical mortality rates of $<5 \%$ $(8-11)$ have re-emphasized the need to consider surgical resection as a therapy with curative potential.

The prognosis for the $85 \%$ of patients with unresectable disease remains dismal. The median survival in this group is 6 months and the primary therapeutic goal is palliation (12, 13). Despite improvement in postoperative outcomes after curative resection, the operative mortality and morbidity for palliative surgery remains high $(14,15)$. Several randomized trials have demonstrated endoscopic biliary endoprosthesis placement to be equal in efficacy to surgical biliary bypass for relief of obstructive jaundice (16-19). In addition, endoscopic palliation is associated with significantly lower morbidity and mortality rates and may shorten the length of hospitalization relative to surgical interventions. Although surgical bypass remains the treatment of choice for duodenal obstruction, this is frequently a terminal event and fewer than $10 \%$ of patients will be candidates for duodenal bypass during the course of the disease (16). Therefore, the majority of patients with unresectable disease can be managed without surgery.

The emergence of nonsurgical means of palliation has underscored the importance of accurate preoperative staging and selection of patients for attempted curative resection. Candidates for attempts at curative surgery should have no evidence of distant metastatic disease, and the tumor should be amenable to complete extirpation based on a lack of invasion into major vascular structures. Computed tomography (CT) and, more recently, laparoscopy have become indispensable for detecting intraabdominal metastatic dis- 
ease (20-25). Because CT is both noninvasive and a reliable means of documenting distant disease, it is the preferred initial procedure in any staging strategy. The detection of tumor invasion of the portal and mesenteric vasculature has traditionally relied on CT and mesenteric angiography (20, 22, 26-31). Endoscopic ultrasound (EUS) has emerged as an innovative pancreatic imaging modality with an accuracy of $>90 \%$ in the local staging of pancreatic cancer (32-37). Although EUS is limited as a means of detecting distant disease, it compares favorably to angiography and CT for defining vascular invasion and tumor size $(33,38,39)$.

There is currently no consensus on the optimal preoperative staging strategy for patients with adenocarcinoma of the pancreas. Furthermore, the impact of individual staging procedures on clinical and economic endpoints remains unclear. Using decision analysis, we investigated the effect of alternative staging strategies employed in clinical practice, incorporating EUS, angiography, and laparoscopy on the clinical outcomes and disease-specific costs in patients with pancreatic cancer.

\section{MATERIALS AND METHODS}

Computer-based models simulating the staging and treatment of patients with adenocarcinoma of the pancreas were created with Decision Maker (Pratt Medical Group, Boston, MA). All patients entering the model were potential surgical candidates with no evidence of unresectable disease on dynamic CT. We chose dynamic as opposed to helical CT scanning because of its widespread availability in the community, its frequent use in screening examinations, and the ongoing debate regarding the magnitude of staging superiority that helical scanning provides (40). We evaluated seven alternative staging strategies representing popular clinical practices:

1. LAP: Patients proceeded directly to laparoscopy and if there was no evidence of intraabdominal dissemination, full surgical exploration was performed with the intent of undertaking a potentially curative resection.

2. ANG-LAP: Angiography was performed as the primary staging procedure, and patients with resectable tumors proceeded to laparoscopy followed by laparotomy for a potentially curative resection as outlined in the LAP strategy.

3. EUS-LAP: EUS was the primary staging procedure; if the tumor seemed resectable, laparoscopy followed by full surgical exploration was performed as in the LAP strategy.

4. LAP-EUS: Laparoscopy was performed as the initial procedure distinct from the planned laparotomy for possible curative resection. When laparoscopy revealed no intraabdominal dissemination, the patient proceeded to EUS and, if the tumor was deemed resectable, full surgical exploration was performed.

5. LAP-ANG: If the initial laparoscopy revealed no intra-
Table 1. Clinical Inputs

\begin{tabular}{lccc}
\hline \multicolumn{1}{c}{ Clinical Variable } & Probability & Range & References \\
\hline Angiography sensitivity* & 0.70 & $0.70-1.00$ & 20,22, \\
Angiography specificity* & 0.91 & $0.70-1.00$ & $26-33$ \\
EUS sensitivity* & 0.91 & $0.70-1.00$ & \\
EUS specificity* & 0.92 & $0.70-1.00$ & $32-39$ \\
Laparoscopy sensitivity $\dagger$ & 0.85 & $0.85-1.00]$ & $21-25$ \\
Laparoscopy specificity ${ }^{\dagger}$ & 1.00 & & $21-23$ \\
Tumor resectability & 0.33 & & \\
Obstructive jaundice & 0.80 & & \\
Duodenal obstruction & 0.10 & & \\
\hline
\end{tabular}

* Detection of locally unresectable disease.

$\dagger$ Detection of intraabdominal dissemination (e.g., peritoneal or hepatic metastasis).

EUS $=$ endoscopic ultrasound.

abdominal dissemination, the patient proceeded to mesenteric angiography. If the tumor was resectable by angiographic criteria, full surgical exploration for a potentially curative resection was performed.

6. ANG-EUS-LAP: Angiography was performed as the primary staging procedure and EUS was performed as a confirmatory procedure only in patients with unresectable disease by angiography. If angiography or EUS demonstrated a resectable tumor, laparoscopy was performed followed by laparotomy when there was no evidence of intraabdominal metastasis.

7. EUS-ANG-LAP: EUS was performed as the primary staging procedure and angiography was performed as a confirmatory procedure only in patients with unresectable disease by EUS. If EUS or angiography demonstrated a resectable tumor, laparoscopy was performed followed by laparotomy when there was no evidence of intraabdominal metastasis. The last two strategies employed the confirmatory procedure as a means of minimizing overstaging and, hence, limiting the allocation of patients with resectable disease to palliative therapy.

Patients with unresectable disease determined by EUS, angiography, or laparoscopy were managed with endoscopic stenting for obstructive jaundice, CT-guided biopsy to confirm a histological diagnosis of adenocarcinoma, and gastrojejunostomy if the patient developed duodenal obstruction. Patients with unresectable disease at laparotomy were managed with surgical palliation, which included pancreatic biopsy, choledochojejunostomy in the setting of obstructive jaundice, and gastrojejunostomy when duodenal obstruction was present or anticipated.

\section{Clinical Inputs}

All assumptions for clinical probabilities were derived from critical analysis of the literature. Performance characteristics for alternative staging procedures and disease natural history are outlined in Table 1.

\section{Cost Inputs}

All cost estimates were based on Medicare reimbursements for our university-based hospital (Table 2). All staging procedures and nonsurgical palliative procedures were as- 
Table 2. Cost Inputs

\begin{tabular}{lcc}
\hline \multicolumn{1}{c}{ Procedure } & Cost* & Range \\
\hline Pancreaticoduodenectomy & $\$ 23,800$ & \\
Choledochojejunostomy & $\$ 16,720$ & \\
Gastrojejunostomy & $\$ 16,160$ & \\
Laparoscopy with biopsy & $\$ 2,880$ & \\
Angiography & $\$ 1,720$ & $\$ 600-2,000$ \\
ERCP with biliary stent & $\$ 1,330$ & \\
CT-guided biopsy & $\$ 760$ & \\
EUS & $\$ 660$ & $\$ 600-2,000$ \\
\hline
\end{tabular}

* Medicare reimbursement.

EUS $=$ endoscopic ultrasound.

sumed to be outpatient procedures, as this is the current standard at the majority of centers. All hospital, professional, pharmaceutical, and pathology fees were included. Indirect costs (e.g., lost productivity) and nonmedical direct costs (e.g., travel, lodging) were not included.

\section{Analysis}

The model simulated the evaluation and initial treatment allocation of 100 patients with pancreatic adenocarcinoma limited to the head of the pancreas on dynamic CT. Favorable clinical outcomes measured included surgical resection for patients with resectable tumors and nonsurgical palliation for patients with unresectable disease. Missed opportunity for cure in patients with resectable disease and surgical exploration for unresectable disease were considered undesirable outcomes. Cost per curative resection was calculated for each of the staging strategies. When one strategy yielded a higher resection rate at an increased cost per curative resection, an incremental cost-effectiveness ratio (additional money spent per additional curative resection) was calculated. Sensitivity analysis examined the impact of varying the performance characteristics for staging techniques (Table 1) and procedural costs (Table 2) over a range of inputs.

\section{RESULTS}

\section{Treatment Allocation}

The distribution of patients into the desirable (e.g., resectable and explored, unresectable and not explored) and undesirable (e.g., resectable and not explored, unresectable
Table 4. Cost Outcomes

\begin{tabular}{lcc}
\hline \multicolumn{1}{c}{ Strategy } & Cost per Patient $(\$)$ & $\begin{array}{c}\text { Cost per Curative } \\
\text { Resection }(\$)\end{array}$ \\
\hline LAP & 18,100 & 54,800 \\
ANG-LAP & 13,400 & 44,600 \\
EUS-LAP & 11,400 & 37,600 \\
LAP-ANG & 15,400 & 51,200 \\
LAP-EUS & 13,800 & 45,300 \\
ANG-EUS-LAP & 14,500 & 44,300 \\
EUS-ANG-LAP & 14,000 & 42,800 \\
\hline
\end{tabular}

Abbreviations as in Table 3.

and explored) clinical categories is represented in Table 3. A strategy employing laparoscopy alone resulted in resection for all patients with resectable tumors, largely because of the requirement of pathological confirmation of distant disease to forestall resection. This level of precision, which eliminated missed opportunities for cure, came at a cost of nearly 40 surgical explorations in patients with unresectable disease per 100 patients staged.

Both ANG-LAP and LAP-ANG appropriately identified 91\% (30 of 33) of individuals with resectable disease; however, $13.2 \%$ of patients staged underwent an unnecessary exploration. Strategies employing EUS followed by laparoscopy (EUS-LAP) or laparoscopy followed by EUS (LAP-EUS) similarly identified a slightly higher percentage (92\%) of patients amenable for cure but resulted in the lowest rate of unnecessary surgical explorations $(5.8 \%$ of patients staged), a greater than 2 -fold reduction relative to angiography-based strategies and a 5-fold reduction relative to laparoscopy alone. The strategies employing a confirmatory procedure when the initial EUS or angiography predicted unresectable disease further increased the resection rate from $92 \%$ (30 of 33) to $99 \%$ (32 of 33); however, this intervention to reduce missed opportunity for cure increased the inappropriate exploration rate nearly 3 -fold from $5.8 \%$ to $15.5 \%$.

\section{Cost Analysis}

Since the goal of staging is the accurate allocation of patients to curative resection and palliative interventions, the economic analysis focused on the cost per curative resection (Table 4). This cost-effectiveness ratio was lowest for the

Table 3. Clinical Outcomes

\begin{tabular}{|c|c|c|c|c|}
\hline \multirow[b]{2}{*}{ Strategy } & \multicolumn{2}{|c|}{ Optimal Treatment Allocation } & \multicolumn{2}{|c|}{ Adverse Treatment Allocation } \\
\hline & $\begin{array}{c}\text { Resectable, } \\
\text { Explored }\end{array}$ & $\begin{array}{l}\text { Unresectable, } \\
\text { Not Explored }\end{array}$ & $\begin{array}{l}\text { Resectable, } \\
\text { Not Explored }\end{array}$ & $\begin{array}{c}\text { Unresectable, } \\
\text { Explored }\end{array}$ \\
\hline LAP & $33.0 \%$ & $27.4 \%$ & $0.0 \%$ & $39.6 \%$ \\
\hline ANG-LAP & $30.0 \%$ & $53.8 \%$ & $3.0 \%$ & $13.2 \%$ \\
\hline EUS-LAP & $30.4 \%$ & $61.2 \%$ & $2.6 \%$ & $5.8 \%$ \\
\hline LAP-ANG & $30.0 \%$ & $53.8 \%$ & $3.0 \%$ & $13.2 \%$ \\
\hline LAP-EUS & $30.4 \%$ & $61.2 \%$ & $2.6 \%$ & $5.8 \%$ \\
\hline ANG-EUS-LAP & $32.8 \%$ & $51.5 \%$ & $0.2 \%$ & $15.5 \%$ \\
\hline EUS-ANG-LAP & $32.8 \%$ & $51.5 \%$ & $0.2 \%$ & $15.5 \%$ \\
\hline
\end{tabular}

ANG = angiography; EUS = endoscopic ultrasound; LAP = laparoscopy. (For additional abbreviations, see Materials and Methods.) 
EUS-LAP strategy at $\$ 37,600$. Reversing the order of staging procedures in the LAP-EUS strategy served only to increase the cost per patient by $\$ 2,300$ without altering the outcomes, yielding a cost per curative resection of $\$ 45,300$. The ANG-LAP strategy increased the cost per patient by $\$ 2,000$ relative to the EUS-LAP strategy, resulting in a cost per curative resection of $\$ 44,600$. Dissection of the expenditures within the model demonstrated that approximately $50 \%$ of this increased cost in the ANG-LAP strategy was attributable to differences in staging procedure costs and $50 \%$ was due to an increase in unnecessary surgical explorations. As with the EUS-based strategy, performing laparoscopy before angiography served only to increase the cost-effectiveness ratio to $\$ 51,200$ without altering outcomes. Laparoscopy alone was the least cost-effective strategy, resulting in a cost per curative resection of $\$ 54,800$, a $46 \%$ increase relative to the EUS-LAP strategy.

Both the EUS-ANG-LAP and ANG-EUS-LAP strategies were associated with increased total cost per patient because of added staging and surgical palliative costs, but the increase in curative resections resulted in intermediate costeffectiveness ratios. The EUS-ANG-LAP strategy was costeffective relative to the ANG-EUS-LAP strategy with a cost per curative resection of $\$ 42,800$.

Because the laparoscopy strategy yielded the highest rate of surgical resections yet required the greatest expenditures, an incremental cost analysis was performed. The additional resections realized in the LAP strategy came at an incremental cost of $\$ 257,000$ per additional curative resection relative to the EUS-LAP strategy and $\$ 2,033,000$ per additional curative resection relative to the EUS-ANG-LAP strategy. Similarly, the improved resection rate in the EUSANG-LAP strategy required $\$ 108,000$ per additional curative resection relative to the EUS-LAP strategy.

\section{Sensitivity Analysis}

The EUS-LAP strategy remained the most cost-effective strategy when the cost of EUS and angiography were varied from $\$ 400$ to $\$ 2000$. The cost per curative resection was also insensitive to alterations of the sensitivity or specificity of EUS and angiography from 0.7 to 1.0 .

\section{DISCUSSION}

Pancreatic cancer is among the most lethal of malignancies, primarily because of the advanced stage at the time of diagnosis. The vast majority of patients have incurable disease, underscoring the importance of preoperative selection of patients for attempted resection. Patients with residual disease after resection have a median survival similar to patients treated with palliative therapy $(8,10,15)$. Furthermore, several randomized trials have demonstrated the equivalent success and lower short-term mortality of nonsurgical palliation of obstructive jaundice relative to surgical interventions (16-18). Therefore, the higher rate of early mortality and morbidity associated with surgical palliation may be avoided in patients with advanced tumors if the preoperative staging strategy accurately identifies patients with unresectable disease. Similarly, to maximize clinical benefit, efforts to avoid unnecessary surgical interventions should not result in an excessive rate of overstaging, thereby denying patients with resectable disease the possibility of cure. Finally, there is also the concern that extensive preoperative imaging protocols increase expenditures without affecting management or outcomes.

Dynamic $\mathrm{CT}$ is a noninvasive means of detecting unresectable disease and should always be the initial imaging procedure when staging adenocarcinoma of the pancreas. Vascular invasion has traditionally been assessed with mesenteric angiography (41), but the emergence of other imaging modalities such as EUS has led to controversy regarding the optimal staging strategy for patients with pancreatic cancer. Recently, laparoscopy has been employed as a more sensitive means of detecting intraabdominal metastasis and is logically employed before a full laparotomy. However, some investigators have suggested laparoscopy as the initial staging procedure after CT as a means of avoiding other invasive imaging procedures such as angiography (23). Others have questioned the need for any specialized imaging procedure to evaluate for the presence of vascular invasion, instead employing CT and laparoscopy alone before surgical exploration. Our model represents the first systematic analysis of potential staging strategies incorporating these imaging procedures.

Because the goal of any staging procedure is to accurately allocate patients to appropriate treatment protocols based on pathological stage at the time of evaluation, our clinical endpoints of interest reflect this intention. The strategy employing EUS followed by laparoscopy (EUS-LAP) yielded the lowest rate of unnecessary surgical explorations and a $2.6 \%$ rate of overstaging. This low rate of overstaging translated into a surgical resection in $92 \%$ of patients with resectable tumors. Both strategies employing angiography as the sole method of detecting vascular invasion increased both undesirable outcomes relative to the EUS strategies. Because of the $100 \%$ specificity of laparoscopy with biopsy, the strategy employing laparoscopy alone maximized the number of resected patients. However, our model demonstrates the cost of this benefit as a 5-fold increase in the number of unnecessary surgical explorations in unresectable patients. Both strategies employing a confirmatory procedure produced a nearly perfect rate of identifying resectable tumors while reducing the number of unnecessary explorations by $61 \%$ relative to laparoscopy alone. Based on these estimated clinical outcomes, strategies incorporating EUS should be favored over strategies incorporating angiography as a means of detecting vascular invasion. Similarly, if minimizing missed opportunities for cure is prioritized, strategies with a confirmatory procedure to evaluate for vascular invasion (e.g., EUS-ANG-LAP) are preferred to laparoscopy alone because of the significant reduction in unnecessary explorations. 
In addition to projecting these clinical endpoints, our analysis provides an estimate of the relative cost-effectiveness of these staging protocols. The traditional strategy of angiography followed by laparoscopy (ANG-LAP) yielded a cost per curative resection of $\$ 44,600$. Substituting EUS for angiography yielded the most cost-effective strategy, lowering the cost per curative resection by $\$ 7000$ to $\$ 37,600$. Approximately one-half of this reduction was attributable to reduced staging costs, and one-half was due to a decrease in unnecessary explorations. Some have proposed laparoscopy as the initial staging procedure before specialized imaging procedures to define vascular invasion (23). In our analysis, both LAP-EUS and LAP-ANG increased the cost per curative resection without altering the clinical outcomes relative to the EUS-LAP and ANG-LAP, respectively. This increased cost is incurred because all patients are subjected up-front to the relatively costly procedure of laparoscopy. Similarly, the EUS-ANG-LAP strategy is preferred over the ANG-EUS-LAP strategy because of the lower cost of the former with identical clinical outcomes. The laparoscopy-alone (LAP) strategy proved to be the least cost-effective. Although this strategy yielded a slightly higher rate of curative resections, the incremental cost of this benefit was $\$ 257,000$ per additional curative resection relative to the EUS-LAP strategy and \$2,033,000 per additional curative resection relative to the EUS-ANGLAP strategy. Therefore, although laparoscopy alone provides a marginal increase in the number of patients undergoing surgical resection, policy makers and clinicians need to consider the increased rate of unnecessary surgical explorations and incremental expenditures before adopting this strategy.

Our analysis cannot be used to select one strategy as the dominant strategy but, rather, provides a framework for physicians, policy makers, and payers to select appropriate staging algorithms given our current understanding of the individual components of staging. In addition, our model allows for the optimal design of future prospective studies designed to clarify the role of specific staging algorithms by avoiding strategies such as the angiography-based strategies, which are clearly deleterious from a clinical and cost perspective.

Our model has several limitations. All decision models contain a level of uncertainty inherent in the clinical and economic assumptions of the model. Sensitivity analysis provides a means of addressing this uncertainty by assigning a range of values to inputs prone to uncertainty. In our analysis we specifically address the importance and relative merits of specialized imaging procedures designed to detect vascular invasion. The cost per curative resection was insensitive to alterations in the cost and a wide range of performance characteristics of EUS and angiography. We examine the cost per curative resection as the primary economic outcome, inasmuch as accurate patient allocation is the primary goal of preoperative staging. It should be emphasized that our definition of curative resection is a patho- logical cure at the time of resection. As the majority of these patients eventually succumb to recurrent disease, these cost estimates grossly underestimate the true cost per cure. An index such as cost per life-year gained would be more useful as a reference with other medical interventions such as the treatment of hypertension; however, our goal was to directly compare alternative staging strategies for pancreatic cancer. Furthermore, obtaining these parameters would require an estimate of survival attributable to accurate versus inaccurate staging, and these figures are currently not available.

Our analysis assumes the payer's perspective and, hence, only direct costs are included. We believe that this provides a conservative estimate of the relative cost-benefit analysis, and institutes a bias in favor of less aggressive staging strategies such as LAP, because the indirect costs are certainly higher for strategies with increased morbidity and mortality from unnecessary surgical explorations.

Finally, we have not included certain innovative imaging modalities such as helical CT, magnetic resonance imaging, and laparoscopic ultrasound, as high quality data on the performance characteristics for pancreatic cancer are still limited. Preliminary data from our institution (42) and others (43) supports the superiority of EUS relative to helical CT for defining locally unresectable disease, and when these results were incorporated into our model, strategies employing EUS remained cost-effective. These data support the strategy employing an initial cross-sectional imaging technique to determine the absence of metastatic disease, followed by a more sensitive technique such as EUS to determine local resectability. The adaptability of decision analysis will permit us to incorporate new information into the analysis as our knowledge of other imaging modalities and interventions for pancreatic cancer advances.

Reprint requests and correspondence: William M. Tierney, M.D., Assistant Professor of Medicine, University of Oklahoma Health Sciences Center, PO Box 26307, Oklahoma City, OK 73126.

Received Sep. 2, 1999; accepted Mar. 10, 2000.

\section{REFERENCES}

1. Parker SL, Tong T, Bolden S, et al. Cancer statistics, 1996. CA Cancer J Clin 1996;65:5-27.

2. Connolly MM, Dawson PJ, Michelassi F, et al. Survival in 1001 patients with carcinoma of the pancreas. Ann Surg 1987; 206:366-70.

3. Trede M. The surgical treatment of pancreatic carcinoma. Surgery 1985;97:28-35.

4. Rosenberg JM, Welch JP, Macaulay WP. Cancer of the head of the pancreas: An institutional review with emphasis on surgical therapy. J Surg Oncol 1985;28:217-21.

5. Tsuchiya R, Noda $T$, Harada N, et al. Collective review of small carcinomas of the pancreas. Ann Surg 1986;203:77-81.

6. Bakkevold KE, Kambestad B. Morbidity and mortality after radical and palliative pancreatic cancer surgery: Risk factors influencing the short-term results. Ann Surg 1993;217:35868. 
7. Gudjonsson B. Cancer of the pancreas: 50 Years of surgery. Cancer 1987;60:2284-303.

8. Nagakawa T, Nagamori M, Futakami F, et al. Results of extensive surgery for pancreatic carcinoma. Cancer 1996;77: $640-5$.

9. Yeo CJ, Cameron JL, Lillemoe KD, et al. Pancreaticoduodenectomy for cancer of the head of the pancreas: 201 Patients. Ann Surg 1995;221:721-33.

10. Trede M, Schwall G, Saeger H. Survival after pancreatoduodenectomy: 118 Consecutive resections without an operative mortality. Ann Surg 1990;211:447-58.

11. Cameron JL, Crist DW, Sitzmann JV, et al. Factors influencing survival after pancreaticoduodenectomy for pancreatic cancer. Am J Surg 1991;161:120-5.

12. Ettinghausen SE, Schwartzentruber DJ, Sindelar WF. Evolving strategies for the treatment of adenocarcinoma of the pancreas. J Clin Gastroenterol 1995;21:48-60.

13. Gudjonsson B, Livstone EM, Spiro HM. Cancer of the pancreas: Diagnostic accuracy and survival statistics. Cancer 1978;42:2494-506.

14. Watanapa P, Williamson RCN. Surgical palliation for pancreatic cancer: Developments during the past two decades. Br J Surg 1992;79:8-20.

15. Lillemoe KD, Sauter PK, Pitt HA, et al. Current status of surgical palliation of periampullary carcinoma. Surg Gynecol Obstet 1993;176:1-10.

16. Smith AC, Dowsett JF, Russell RCG, et al. Randomised trial of endoscopic stenting versus surgical bypass in malignant low bile duct obstruction. Lancet 1994;344:1655-60.

17. Andersen JR, Sorensen SM, Kruse A, et al. Randomised trial of endoscopic endoprosthesis versus operative bypass in malignant obstructive jaundice. Gut 1989;30:1132-5.

18. Shepherd HA, Royle G, Ross APR, et al. Endoscopic biliary endoprosthesis in the palliation of malignant obstruction of the distal common bile duct: A randomized trial. Br J Surg 1988; 75:1166-8.

19. Siegel JH, Snady H. The significance of endoscopically placed prostheses in the management of biliary obstruction due to carcinoma of the pancreas: Results of nonoperative decompression in 277 patients. Am J Gastroenterol 1986;81:634-41.

20. Freeny PC, Marks WM, Ryan JA, et al. Pancreatic ductal adenocarcinoma: Diagnosis and staging with dynamic CT. Radiology 1988;166:125-33.

21. Warshaw AL, Tepper JE, Shipley WU. Laparoscopy in the staging and planning of therapy for pancreatic cancer. Am J Surg 1985;151:76-80.

22. Warshaw AL, Gu Z, Wittenberg J, et al. Preoperative staging and assessment of resectability of pancreatic cancer. Arch Surg 1990;125:230-3.

23. Castillo CF, Rattner DW, Warshaw AL. Further experience with laparoscopy and peritoneal cytology in the staging of pancreatic cancer. Br J Surg 1995;82:1127-9.

24. John TG, Greig JD, Carter DC, et al. Carcinoma of the pancreatic head and periampullary region: Tumor staging with laparoscopy and laparoscopic ultrasonography. Ann Surg 1995;221:156-64.

25. Bemelman WA, DeWit LT, Van Delden OM, et al. Diagnostic laparoscopy combined with laparoscopic ultrasonography in staging of cancer of the pancreatic head region. Br J Surg 1995;82:820-4.
26. Mackie CR, Noble HG, Cooper MJ, et al. Prospective evaluation of angiography in the diagnosis and management of patients suspected of having pancreatic cancer. Ann Surg 1979;189:11-7.

27. Howard TJ, Chin AC, Strieb EW, et al. Value of helical computed tomography, angiography, and endoscopic ultrasound in determining resectability of periampullary carcinoma. Am J Surg 1997;174:237-41.

28. Murugiah M, Windsor JA, Redhead DN, et al. The role of selective visceral angiography in the management of pancreatic and periampullary cancer. World J Surg 1993;17:796800.

29. Appleton GVN, Bathurst NCG, Virjee J, et al. The value of angiography in the surgical management of pancreatic disease. Ann R Coll Surg Engl 1989;71:92-6.

30. Hemmigsson A, Jacobson G, Lindgren PG, et al. Radiologic assessment of resectability of carcinoma of the head of the pancreas. Acta Radiol Diagnosis 1982;23:127-30.

31. Jafri SZ, Aisen AM, Glazer GM, et al. Comparison of CT and angiography in assessing resectability of pancreatic carcinoma. AJR 1984;142:525-9.

32. Snady H, Bruckner H, Siegel J, et al. Endoscopic ultrasound criteria of vascular invasion by potentially resectable pancreatic tumors. Gastrointest Endosc 1994;40:326-33.

33. Rosch T, Braig C, Gain T, et al. Staging of pancreatic and ampullary carcinoma by endoscopic ultrasonography. Gastroenterology 1992;102:188-99.

34. Tio TL, Tytgat GN, Cikot RJ, et al. Ampullopancreatic carcinoma: Preoperative TNM classification with endosonography. Radiology 1990;175:455-61.

35. Palazzo L, Roseau G, Gayet B, et al. Endoscopic ultrasonography in the diagnosis and staging of pancreatic adenocarcinoma. Endoscopy 1993;25:143-50.

36. Nakaizumi A, Uehara H, Iishi H, et al. Endoscopic ultrasound in diagnosis and staging of pancreatic cancer. Dig Dis Sci 1995;40:696-70.

37. Muller MF, Meyenberger C, Bertschinger P, et al. Pancreatic tumors: Evaluation with endoscopic US, CT, and MR imaging. Radiology 1994;190:745-51

38. Giovanni M, Steitz JF. Endoscopic ultrasound with linear-type echoendoscope in the evaluation of 94 patients with pancreaticobiliary disease. Endoscopy 1994;26:579-85.

39. Brugge WR, Lee MJ, Kelsey PB, et al. The use of EUS to diagnose malignant portal venous system invasion by pancreatic cancer. Gastrointest Endosc 1996;43:561-7.

40. Hiroki T, Hauptmann E, Traverso L, et al. How accurate is helical computed tomography for clinical staging of pancreatic cancer. Am J Surg 1999; 177:428-32.

41. Cameron JL. The current management of carcinoma of the head of the pancreas. Annu Rev Med 1995;46:361-70.

42. Tierney WM, Carpenter SL, Bansal R, et al. Accuracy and economic impact of helical CT and endoscopic ultrasound in the staging of ampullopancreatic tumors. Gastrointest Endosc 1997;45:AB183.

43. Gress F, Ciaccia D, Kiel J, et al. Endoscopic ultrasound versus sprial computed tomography for staging pancreatic, biliary and ampulary tumors: A prospective comparison. Gastroeintest Endosc 1997;45:AB173. 